\title{
A comparison of the burden of lung cancer attributable to tobacco exposure in China and the USA
}

\author{
Xiaomei $\mathrm{Wu}^{1}$, Bo $\mathrm{Zhu}^{2}$, Shuang $\mathrm{Xu}^{3}$, Yong $\mathrm{Liu}^{4}$, Yifei $\mathrm{Bi}^{5}$, Baosen $\mathrm{Zhou}^{1}$ \\ ${ }^{1}$ Department of Clinical Epidemiology and Center of Evidence Based Medicine, The First Hospital of China Medical University, Shenyang, China; \\ ${ }^{2}$ Department of Cancer Prevention and Treatment, Cancer Hospital of China Medical University/Liaoning Cancer Hospital \& Institute, Shenyang, \\ China; ${ }^{3}$ Library of China Medical University, Shenyang, China; ${ }^{4}$ Periodontology and Preventive Dentistry, Saarland University, Saarbrücken, \\ Germany; ${ }^{5}$ College of Foreign Languages, University of Shanghai for Science and Technology, Shanghai, China \\ Contributions: (I) Conception and design: X Wu, B Zhu, B Zhou; (II) Administrative support: B Zhou; (III) Provision of study materials or patients: X \\ Wu, B Zhu; (IV) Collection and assembly of data: B Zhu, S Xu; (V) Data analysis and interpretation: X Wu, B Zhu, Y Liu, Y Bi; (VI) Manuscript \\ writing: All authors; (VII) Final approval of manuscript: All authors. \\ Correspondence to: Baosen Zhou. Department of Clinical Epidemiology and Center of Evidence Based Medicine, The First Hospital of China Medical \\ University, No.155 Nanjing Bei Street, Heping District, Shenyang 110001, China. Email: bszhou@cmu.edu.cn.
}

Background: Tobacco exposure (TE) is the major contributor to lung cancer mortality worldwide. This study aims to clarify the possible reasons underlying the long-term trends and differences in lung cancer mortality attributable to TE in China and the United States of America (USA).

Methods: Lung cancer mortality data from China and the USA were obtained from the Global Burden of Disease (GBD) Study 2017. Joinpoint regression analysis was used to assess the magnitude and direction of trends from 1990 to 2017, and the age-period-cohort model (APCM) was used to analyze the temporal trends of lung cancer mortality by age, period, and cohort.

Results: For lung cancer attributable to smoking, the age-standardized mortality rates (ASMRs) exhibited an upward trend in China and a downward trend in the USA. The overall net drifts per year were $0.72 \%$ for China and $-3.03 \%$ for the USA, and the local drift values in China and the USA increased by age group. All cohort/period relative risks (RRs) increased in China but decreased in the USA. The longitudinal age curves increased rapidly in China, whereas those in the USA were rose gradually. For lung cancer attributable to secondhand smoke, the ASMRs showed a downward trend in both China and the USA. The overall net drifts per year were $-0.48 \%$ for China and $-3.97 \%$ for the USA, and the local drift values in China and the USA also increased by age group. Cohort/period RRs decreased in the USA, and fluctuated in China. The longitudinal age curve trends in China and the USA were similar to those of smoking.

Conclusions: The dangers of TE in China remain serious. The rates of lung cancer mortality attributable to TE have increased, especially in older individuals. We recommend that China strengthens the implementation of effective targeted tobacco control policies and other interventions to reduce the burden of lung cancer.

Keywords: Tobacco exposure (TE); lung cancer; age-period-cohort analysis

Submitted Jan 23, 2020. Accepted for publication Aug 28, 2020.

doi: $10.21037 /$ atm-20-996

View this article at: http://dx.doi.org/10.21037/atm-20-996 


\section{Introduction}

In recent decades, lung cancer has surpassed all other cancers for morbidity and mortality worldwide. According to the Global Cancer Observatory (GLOBOCAN) 2018, there were approximately 2.1 million new cases of lung cancer globally, accounting for $11.6 \%$ of all new cancer patients in that year, and approximately 1.8 million deaths, accounting for $18.4 \%$ of all cancer-related mortality (1). More than one-third of all newly diagnosed lung cancer cases occurred in China. According to the age-standardized incidence rate by world standard population (ASIRW), the incidence of lung cancer in China is 36.09/100,000, the cumulative incidence is $4.42 \%$ ( $0-74$ years), the mortality rate is $28.41 / 100,000$, and the cumulative mortality rate is $3.34 \%$ (0-74 years). Also, the rates of morbidity and mortality of lung cancer are higher in males than in females (2). As in China, lung cancer is the leading cause of cancer death among both men and women in the United States of America (USA), accounting for one-quarter of all cancer-related mortality (3). However, in recent years, the incidence of lung cancer in the USA has continued to decline, with the rate of decline being twice as rapid in men as in women. The lung cancer mortality rate decreased by $48 \%$ from 1990 to 2016 among males, and by $23 \%$ from 2002-2016 among females (3). Thus, identifying the controllable risk factors that led to a decrease in lung cancer mortality trends in the USA is crucial.

Tobacco exposure (TE) primarily refers to active smoking and second-hand smoke. Each year, almost 7 million people died from TE globally, with approximately 6 million of these being active tobacco users and an estimated 890,000 being non-smokers exposed to secondhand smoke from 2008-2016 (4,5). Smoking remains the major etiological factor, contributing to $87 \%$ of all lung cancer deaths (6). The different phases of the smoking epidemic have affected the patterns of lung cancer incidence and mortality (7). Smoking has a causal relationship with lung cancer, and secondhand smoke has also been associated with an increased risk of lung cancer in non-smokers (8). In 2017, smoking was ranked highest for cancer burden in China, with secondhand smoke was ranked among the top 10 risk factors (9). Therefore, in our study, we analyzed both smoking and secondhand smoke to comprehensively reflect the impact of TE on lung cancer.

In China, smoking habits and behaviors among the population have become more westernized (10). In 2019, the World Health Organization (WHO) reported estimates for the prevalence of smoking in all countries in response to the global tobacco epidemic (11). China was reported to have a higher prevalence of smoking and higher levels of exposure to second-hand smoke than the USA. Additionally, China had experienced a smaller decline in the prevalence of smoking.

In this study, we compared lung cancer mortality attributable to TE in China and the USA from 1990 to 2017. We collected data from the Global Burden of Disease (GBD) Study 2017, and used the age-period-cohort model (APCM) with an intrinsic estimator (IE) algorithm to analyze the temporal trends of lung cancer mortality based on the independent effects of chronological age, time period, and birth cohort. There are currently a small number of studies on the differences between age groups; however, they all lack comprehensive analyses of the possible reasons underlying the long-term trends of lung cancer mortality attributable to TE.

We present the following article in accordance with the MDAR checklist (available at: http://dx.doi.org/10.21037/ atm-20-996).

\section{Methods}

\section{Data source}

The GBD 2017 study included data on 84 behavioral, environmental, occupational, and metabolic risks or clusters of risks, 282 causes of mortality, 359 diseases and injuries, and Health Adjusted Life Expectancy (HALE) for 195 countries and territories from 1990 to 2017. The study quantified the impact of hundreds of diseases, injuries, and risk factors in countries around the world, and to date is the most comprehensive effort to measure epidemiological trends worldwide. In the GBD2017 study, four elements were considered in assessing the burden of disease including: the metric of burden being assessed (such as a number of deaths), the exposure levels for a risk factor, the relative risk (RR) of a given outcome due to exposure, and the counterfactual level of risk factor exposure. Data are available at the GBD Data Tool repository and can be accessed at http://ghdx.healthdata.org/gbd-results-tool (12).

To analyze the status of lung cancer mortality attributable to TE in China and the USA, the relevant data on lung cancer mortality were extracted including annual mortality cases, the age-standardized mortality rate (ASMR) by year, and the age-group mortality rate. For the purposes of our study, lung cancer (C33-C34) included cancers of 
the trachea, bronchus, and lungs in accordance with the International Statistical Classification of Diseases and Related Health Problems (ICD)-10.

\section{Statistical analyses}

Since the age structure of Chinese and American populations is different, it was necessary to perform standardization to compare mortality trends. ASMR was used to estimate the trend. The ASMR (per 100,000 people) is calculated by the direct method, which is the sum of the age-specific rates ( $a_{i}$, where $I$ denotes the $i^{t h}$ age class) and the number of persons (or weight, $w_{i}$ ) in the same age subgroup (i) of the chosen reference standard population, divided by the sum of standard population weights. The formula used is as follows:

$$
\mathrm{ASR}=\frac{\sum_{i=1}^{A} a_{i} w_{i}}{\sum_{i=1}^{A} w_{i}} \times 100,000
$$

Joinpoint software (Version 4.7.0.0) was used to calculate the annual percentage change (APC) and the corresponding 95\% confidence intervals (CIs) to assess the magnitude and direction of trends over time for mortality rate by joinpoint regression analysis. Joinpoint software calculated the trend data with the natural logarithm of age-standardized rates as the dependent variable $(y)$ and the calendar year as the independent variable $(x)$, and fitted the linear model: $y=\alpha$ $+\beta x+\varepsilon,(\alpha$ : constant term, $\beta$ : regression coefficient, and $\varepsilon$ : random error term). APC was estimated by the regression coefficient, expressed as a percentage: $A P C=100 \times\left(e^{\beta}-1\right)$. The tests of significance used a Monte Carlo permutation method, and the overall asymptotic significance level was maintained through a Bonferroni correction (13). Three joinpoints were used to uniformly detect four different trends in order to investigate the trend of mortality rate of lung cancer attributable to TE in different periods. The population etiologic fraction (PEF), which indicates the risk of TE in the population, was determined by dividing the mortality attributable to TE by the total mortality. Correlation analysis was used to evaluate the strength of the relationship between lung cancer attributable to TE and total lung cancer.

To assess death risks in the population in a particular year and the accumulation of health risks since birth, we used the APCM. The APCM allows for the analysis of the independent effects of age, period, and cohort on temporal trends in lung cancer mortality attributable to $\mathrm{TE}$, and assessment of the relative contribution of both TE and tobacco controls. Age effect represents the change in the mortality rate of diseases caused by physiological and pathological changes with age; period effect represents the change in the mortality rate of diseases caused by various events over time; cohort effect represents the change in the mortality rate of diseases caused by lifestyle or different exposure risk factors. The APCM provides a useful parametric framework that complements standard nonparametric descriptive methods. In the APCM, the collected data needed to be converted to successive 5-year age groups and consecutive 5 -year periods.

Since the GBD dataset did not provide successive 5-year age groups for smokers under 29 or over 95 years of age, the mortality rates of lung cancer attributable to smoking were recorded into successive 5 -year age groups (from 30-34 to 90-94 years) and consecutive 5-year periods (from 1990-2017). Also, since the GBD dataset did not provide successive 5 -year age groups under 24 or over 95 years of age for secondhand smoke, the mortality rates of lung cancer attributable to secondhand smoke were recorded into successive 5-year age groups (from 25-29 to 9094 years) and consecutive 5 -year periods (from 1990-2017). The general linear model was used to analyze the slope of the period/cohort RRs. The APCM was performed using the "apc" package in $\mathrm{R}$ statistical software ( $\mathrm{R}$ version 3.5.1).

The study was conducted in accordance with the Declaration of Helsinki (as revised in 2013). All our research data are obtained from GBD 2017, the website was http://ghdx.healthdata.org/gbd-results-tool. GBD 2017 was publicly available for free use. The ethical approval was not required.

\section{Results}

\section{Temporal trends in the ASMRs of lung cancer attributable to TE from 1990 to 2017}

In our study, for lung cancer attributable to smoking, there were 7,115,511 lung cancer cases in China $(6,327,727$ males and 787,784 females) and 3,588,241 lung cancer cases in the USA $(2,153,434$ males and 1,434,807 females) aged between 30 and 94 years old from 1990 to 2017. The ASMRs of lung cancer attributable to smoking for both sexes in China were lower than those in the USA from 1990 to 2013, with no significant difference in the ASMRs between China and the USA from 2014 to 2017 (Figure 1A). In this period, the ASMRs in China increased significantly [average annual percentage change (AAPC): 1.8\%; 95\% CI: $1.3 \%, 2.2 \%$ ], 


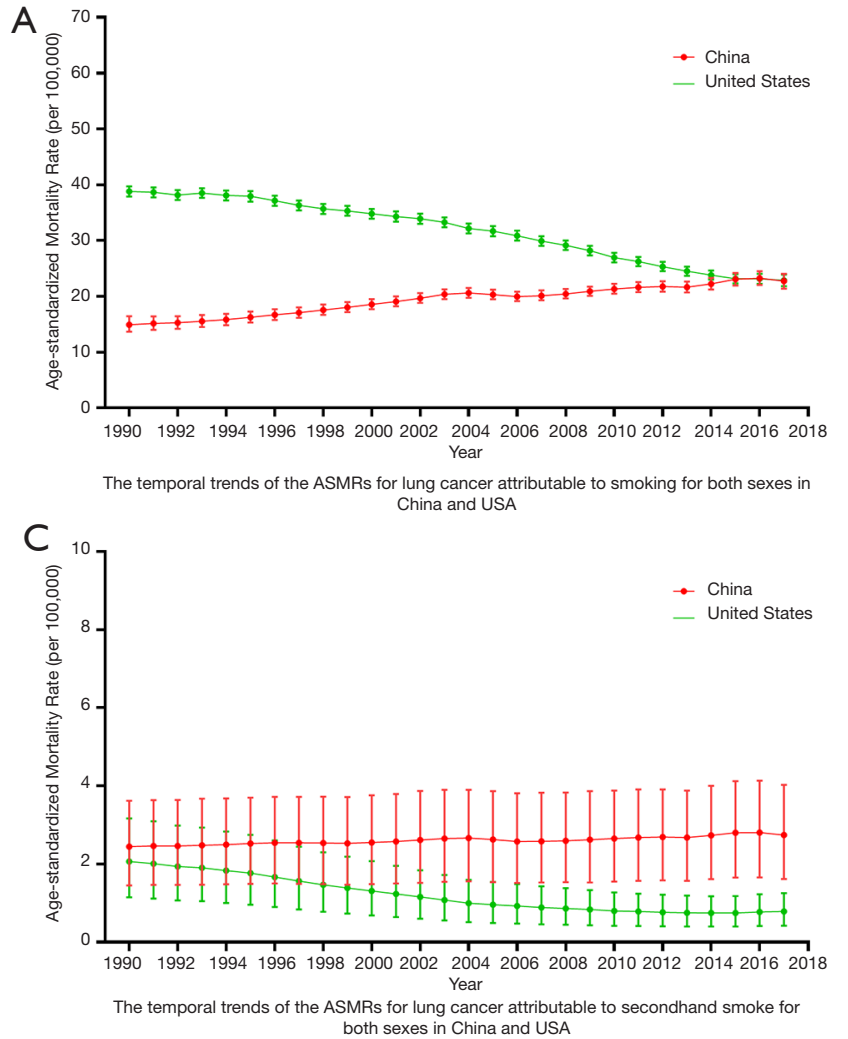

Figure 1 The temporal trends of the age-standardized mortality rates (ASMRs) for lung cancer attributable to tobacco exposure for both sexes (by sex) in China and the USA.

and decreased substantially in the USA (AAPC: $-2.0 \%$; $95 \%$ CI: $-2.2 \%,-1.8 \%$ ) (Table 1). The temporal trends of the ASMRs for lung cancer attributable to smoking by sex in China and the USA are shown in Figure 1B. For males, the ASMRs in China were lower than those in the USA from 1990 to 2007. However, after 2010, the ASMRs in China were considerably higher than those in the USA. For females, the ASMRs in China were consistently lower than those in the USA from 1990 to 2017. The changes in the ASMRs in both countries were similar for both sexes (Table 1).

For lung cancer attributable to second-hand smoke, there were 936,960 lung cancer cases in China (485,061 males and 451,899 females) and 128,511 lung cancer cases in the USA (79,217 males and 49,294 females) aged between 25 and 94 years from 1990 to 2017. Prior to 2007, there were no significant differences between China and the USA in the ASMRs of both sexes for lung cancer attributable to secondhand smoke. However, after 2008, the ASMRs in China were higher than those in the USA (Figure 1C,D). In this period, the ASMRs in China increased significantly
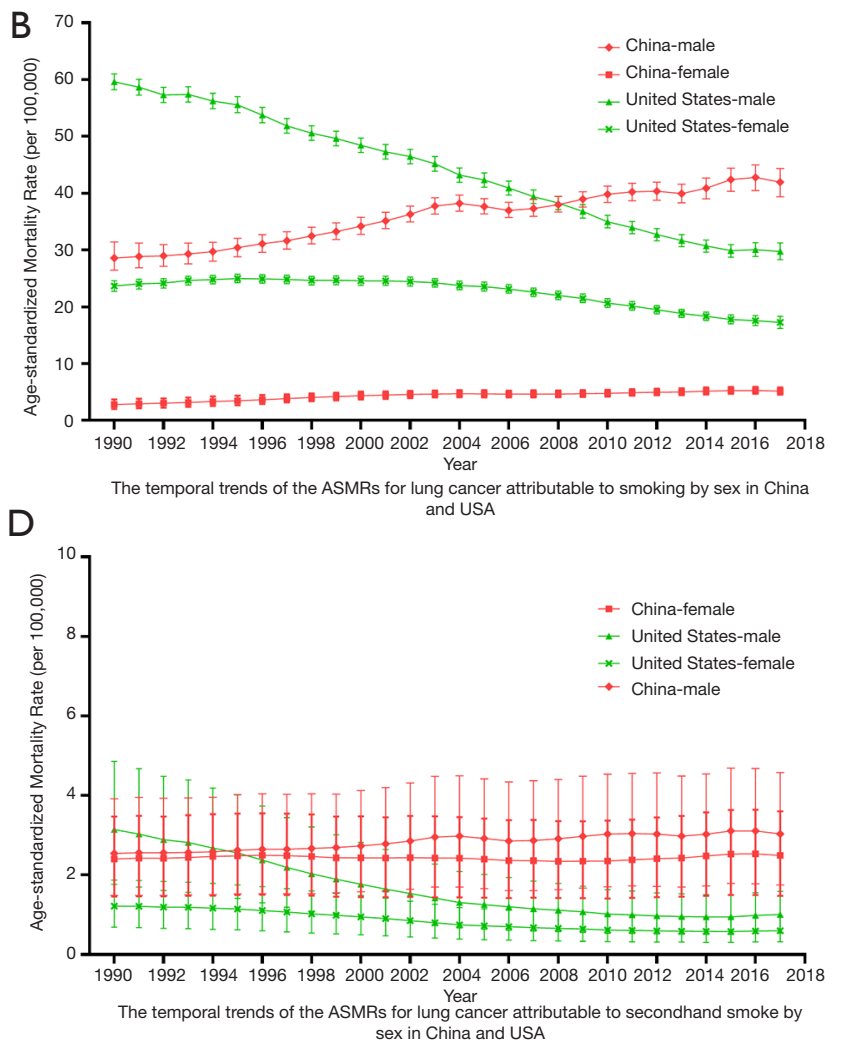
(AAPC: $0.5 \% ; 95 \%$ CI: $0.2 \%, 0.8 \%$ ), but decreased markedly in the USA (AAPC: $-3.7 \% ; 95 \%$ CI: $-4.1 \%$, $-3.3 \%$ ) (Table 1).

Prior to 2010, there was no significant difference between China and the USA in the ASMRs of males for lung cancer attributable to secondhand smoke. However, after 2010, the ASMRs in China were higher than those in the USA. For females, there were no significant differences between China and the USA in the ASMRs of lung cancer attributable to secondhand smoke before 2002. After 2002, the ASMRs in China were higher than those in the USA. The changes of the ASMRs in both countries were similar for both sexes (Table 1).

\section{APCM analysis of the mortality rate of lung cancer attributable to TE from 1990 to 2017}

Figure 2 shows the net and local drifts for the mortality rates of lung cancer attributable to smoking in China and the USA, respectively. Figure $2 A$ shows that the overall net drifts per year were $0.72 \%$ (95\% CI, $0.48 \%$ to $0.96 \%$ ) 


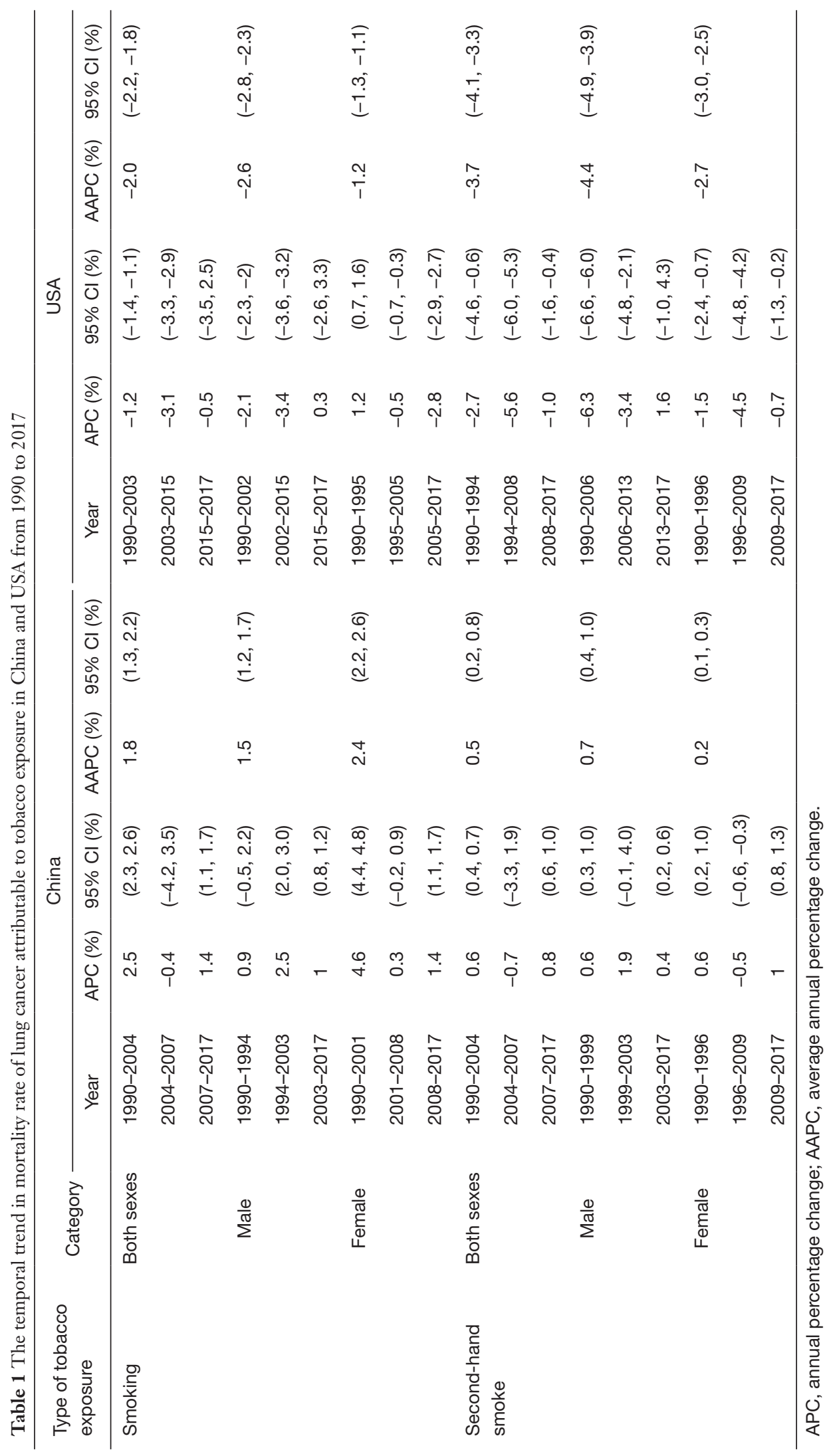



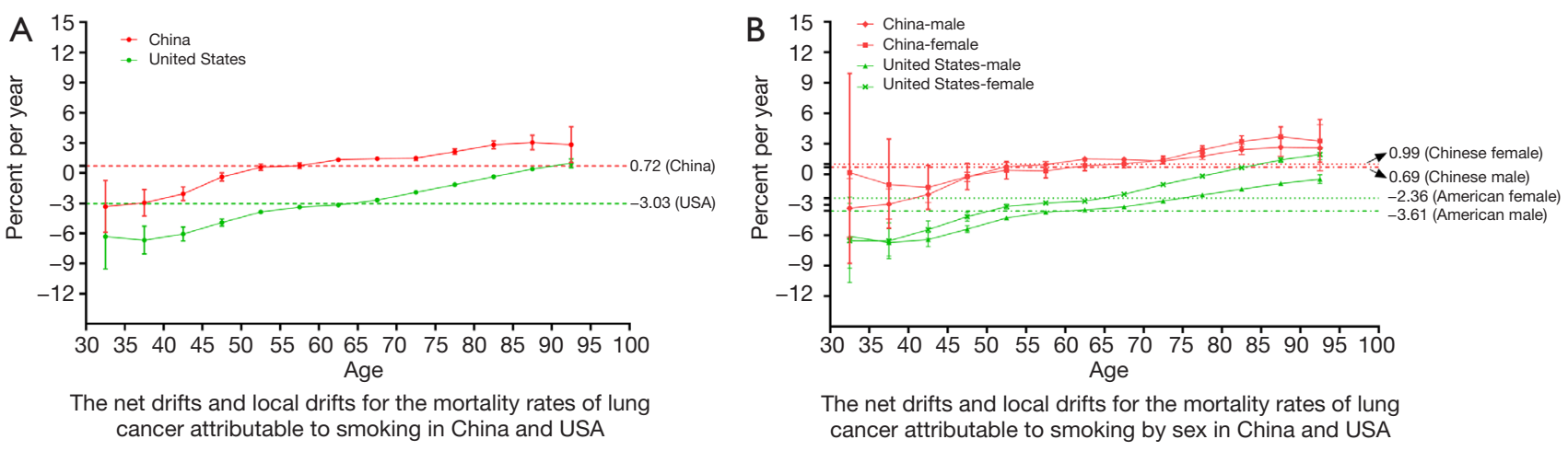

Figure 2 The net drifts and local drifts for the mortality rates of lung cancer attributable to smoking for both sexes (by sex) in China and the USA.

for China and $-3.03 \%$ (95\% CI, $-3.25 \%$ to $-2.82 \%$ ) for the USA, and the local drift values in China and the USA increased by age group. Figure $2 B$ shows the overall net drifts per year by sex in China and the USA. The results were similar for both sexes, and the local drift values in China and the USA also increased by age group.

Figure $3 A, B$ shows the longitudinal age curves for the mortality rates of lung cancer attributable to smoking in China and the USA. In the same birth cohort, the mortality rate in China increased gradually, reaching a peak in the 90-94 age group. However, the mortality rate in the USA reached a peak in the $80-84$ age group and then declined. By country, the trend was similar for both sexes.

Figure $3 C, D$ shows the trend of the estimated period RRs in China and the USA. The period RRs in China increased from 1990 to 2017, while the period RRs in the USA decreased during this time. Compared to the USA, period RRs in China were lower before 2002, but higher after 2002. By country, the trend was similar for both sexes.

Figure $3 E, F$ shows the trend of the estimated cohort RRs in China and the USA. The cohort RRs in China were on an upward trend before 1955, and fluctuated in the period between 1960 and 1985. Meanwhile, the cohort RRs in the USA were consistently on a downward trend. Compared to the USA, the cohort RRs in China were lower prior to 1940, but higher after 1940 .

Figure 4 shows the net and local drifts for the mortality rates of lung cancer attributable to secondhand smoke in China and the USA, respectively. Figure $4 A$ shows that the overall net drifts per year were $-0.48 \%$ (95\% CI, $-0.69 \%$ to $-0.27 \%$ ) for China and $-3.97 \%$ (95\% CI, $-4.26 \%$ to $-3.67 \%)$ for the USA. The local drift values in China and the USA increased by age group. Figure $4 B$ shows the overall net drifts for each year by sex in China and the USA. The results were similar for both sexes, and the local drift values in China and the USA also increased by age group.

Figure $5 A, B$ shows the longitudinal age curves for the mortality rates of lung cancer attributable to secondhand smoke in China and the USA. In the same birth cohort, the mortality rate in China increased gradually, reaching a peak in the 90-94 age group. However, the mortality rate in the USA reached a peak in the 65-69 age group and then declined. By country, the trend was similar for both sexes.

Figure 5C,D shows the trend of the estimated period RRs in China and the USA. The period RRs in China and the USA decreased from 1990 to 2017. Compared to the USA, the period RRs in China were lower prior to 2002 but were higher after 2002. By country, the trend was similar for both sexes.

Figure 5E,F shows the trend of the estimated cohort RRs in China and the USA. The cohort RRs in China were on an upward trend before 1950, and on a downward trend from 1955 to 1990. Meanwhile, the cohort RRs in the USA were on a consistently downward trend. Compared to the USA, the cohort RRs in China were lower prior to 1940 but higher after 1940 .

\section{Discussion}

China, with over 350 million smokers, is the largest tobacco producer and consumer in the world (14). Shi et al. found that the total estimated expenditure of lung cancer attributable to smoking in China in 2015 was US\$5.249 billion, accounting for $0.79 \%$ of China's total healthcare expenditure. This expenditure is likely to increase given the current trends in lung cancer (10). In China, the economic burden of lung cancer attributable to 


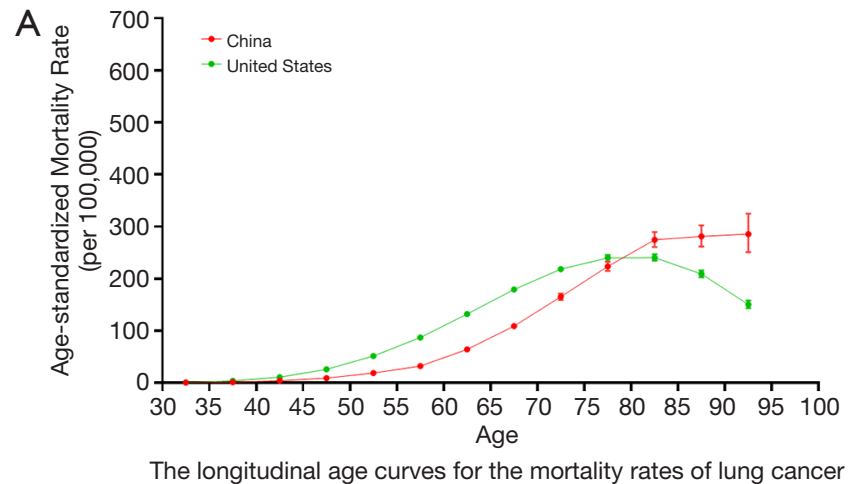
attributable to smoking in China and USA

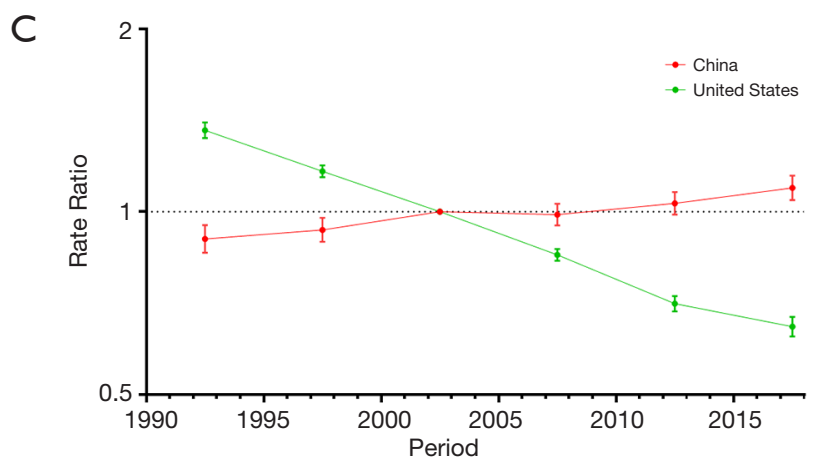

The period RRs for the mortality rates of lung cancer attributable to smoking in China and USA

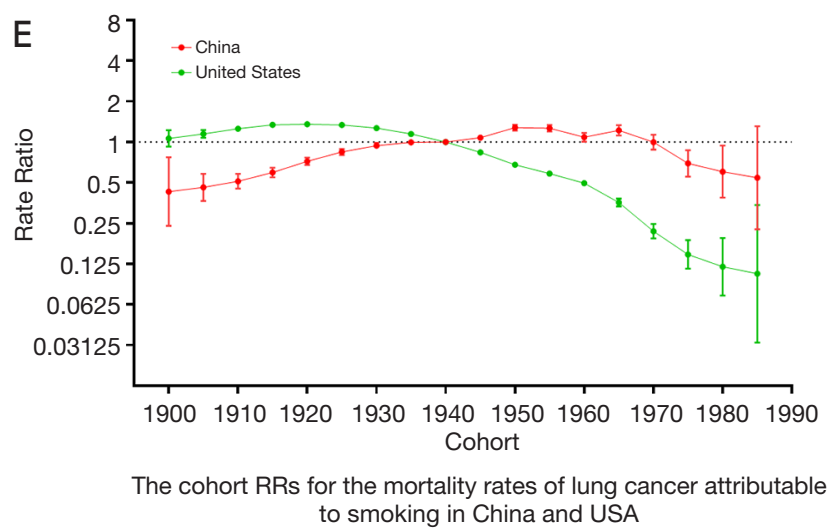

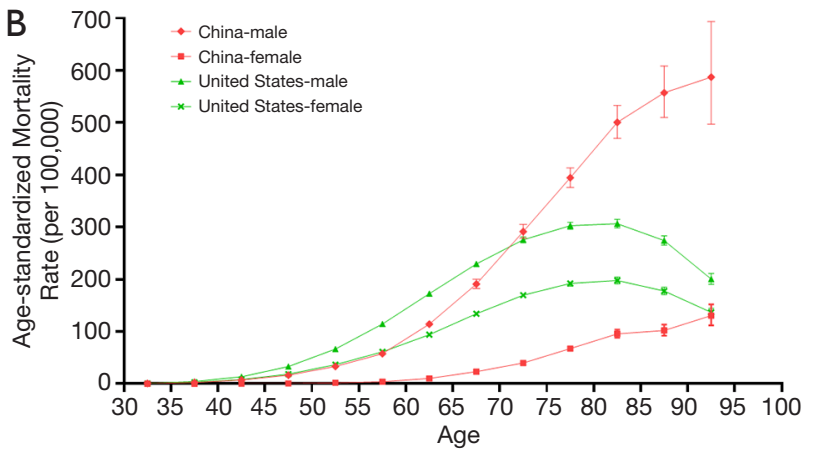

The longitudinal age curves for the mortality rates of lung cancer attributable to smoking by sex in China and USA

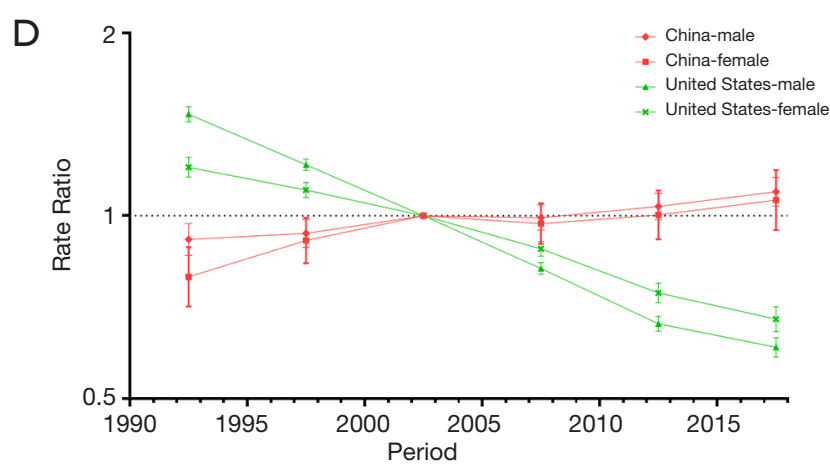

The period RRs for the mortality rates of lung cancer attributable to smoking by sex in China and USA

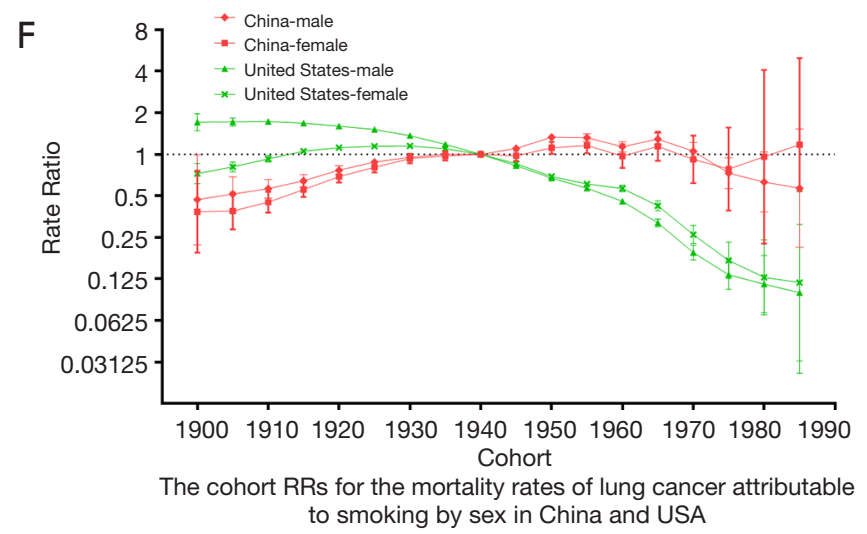

Figure 3 The age-period-cohort results in the mortality rate of lung cancer attributable to smoking for both sexes (by sex) from 1990 to 2017.

smoking in 2015 was considerably greater than that of all other cancers in 2008, accounting for more than one-third of the direct expenditure for lung cancer (15-17). In our study, ASMR trends reflect the shifting patterns of disease in a population and provide clues about the changing factors. For lung cancer cases attributable to smoking, the
ASMR in China was twice that of the USA from 1990 to 2017, while for lung cancer cases attributable to secondhand smoke, the number in China was seven times that of the USA. The trends of ASMR attributable to TE increased in China and decreased in the USA, which was similar to the trends of all lung cancers (see Table S1). PEFs in smoking 


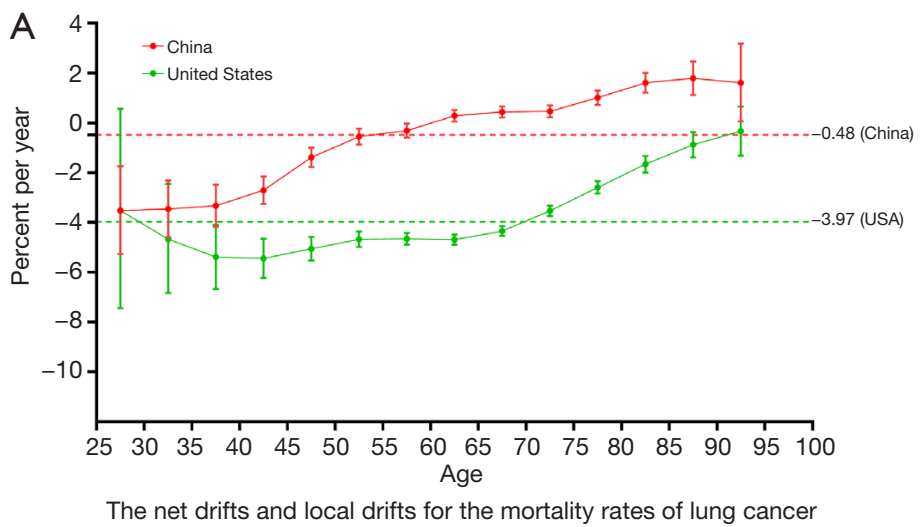
attributable to secondhand smoke in China and USA

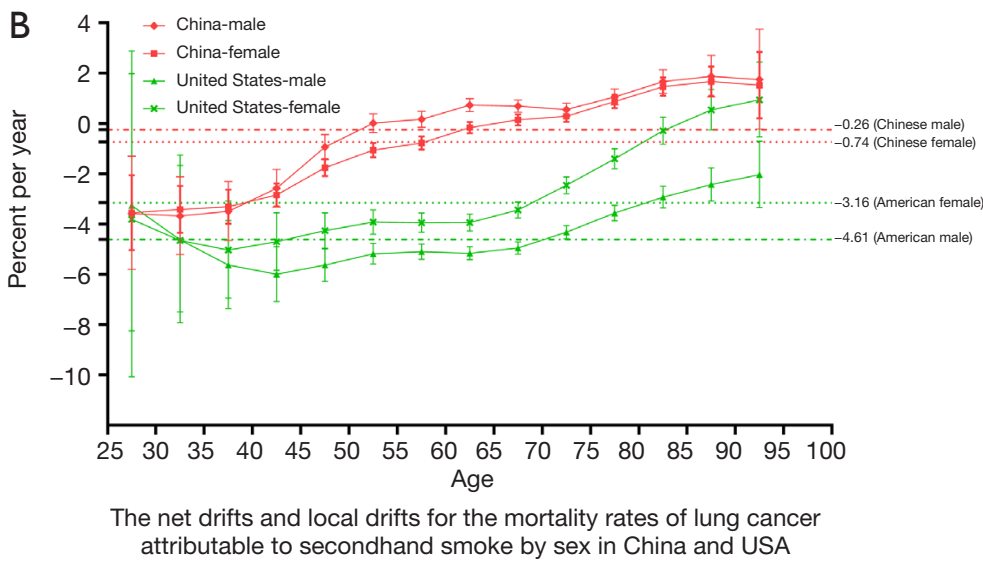

Figure 4 The net drifts and local drifts for the mortality rates of lung cancer attributable to secondhand smoke for both sexes (by sex) in China and the USA.

increased from $52.7 \%$ to $62.7 \%$ in China and decreased from $82.7 \%$ to $66.5 \%$ in the USA. PEFs in secondhand smoke decreased both in China and the USA (see Table S2). Smoking was the primary contributing factor to lung cancer attributable to TE.

In order to identify the reasons for the different trends in lung cancer mortality attributable to TE in the two countries, we used an APCM for analysis. The APCM could analyze the independent effects of age, period, and cohort on temporal trends in lung cancer mortality attributable to TE, and assess the relative contribution of both TE and tobacco controls. Our study sought to analyze differences in the trends of lung cancer mortality attributable to TE between China and the USA during the period 1990-2017. For smoking, the overall net drift in China was above 0, while in the USA it was below 0 . This suggests that lung cancer mortality attributable to smoking had exhibited an upward trend in China and a downward trend in the USA.
In the local drifts, lung cancer mortality in China increased in the 50-94 age group, whereas in the USA, it increased in the 85-94 age group. This demonstrates that the 50-94 age cohort was the main contributor to the rising morality rate of lung cancer attributable to smoking in China. For secondhand smoke, the overall net drift in both China and the USA was below 0 , suggesting that lung cancer mortality attributable to secondhand smoke exhibited a downward trend in both countries, although the decline was less significant in China compared to the USA. In the local drifts, lung cancer mortality in China increased in the 60-94 age group, and decreased in all age groups in the USA. This suggests that people in the 60-94 age cohort maybe at an increased risk from secondhand smoke. We also found that the longitudinal age curves in both countries increased with age. The higher mortality rates among older age groups is consistent with relevant WHO studies (11), and also with age-related decline in immunity and increased risk of 

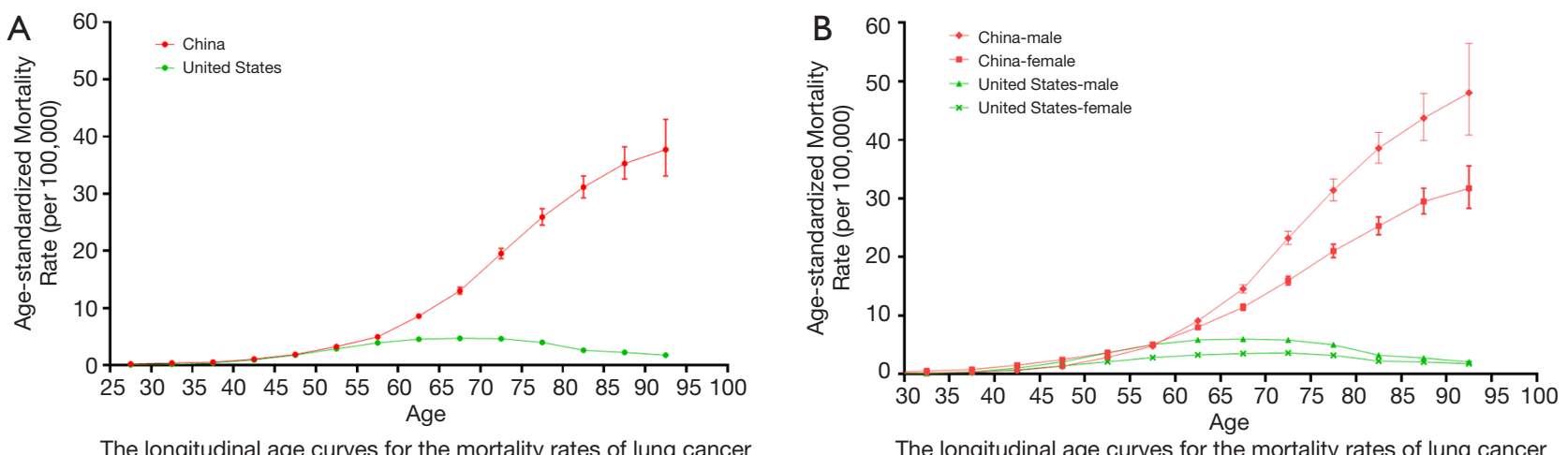

The longitudinal age curves for the mortality rates of lung cancer attributable to secondhand smoke in China and USA
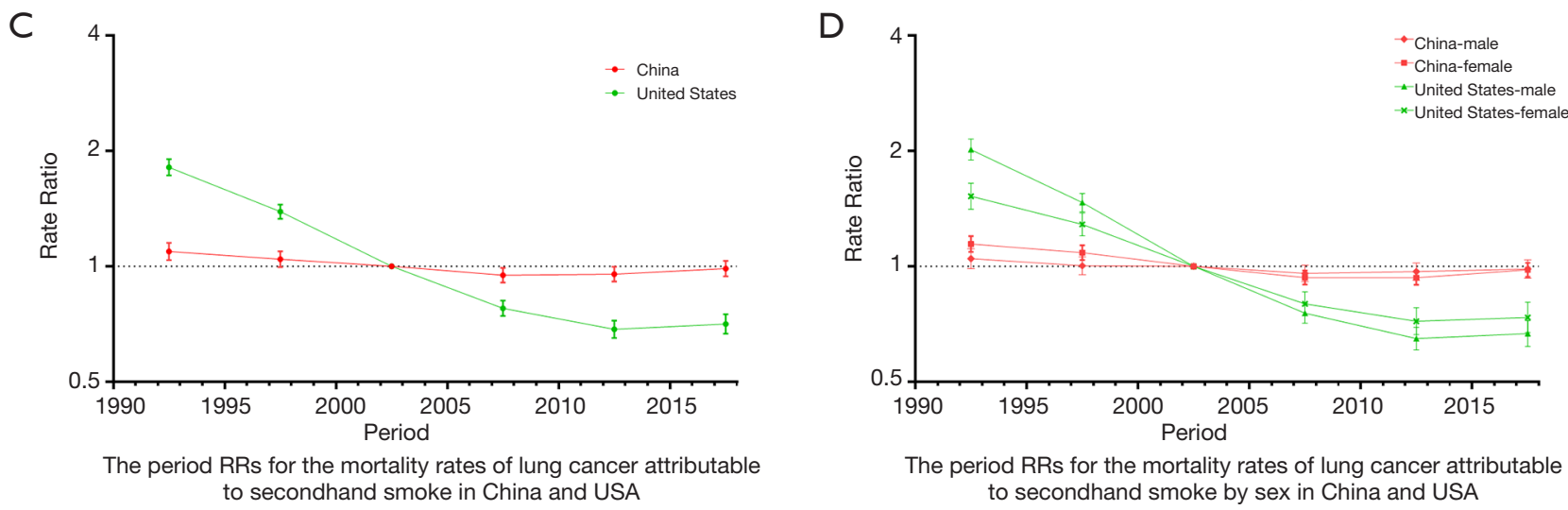

The period RRs for the mortality rates of lung cancer attributable to secondhand smoke by sex in China and USA
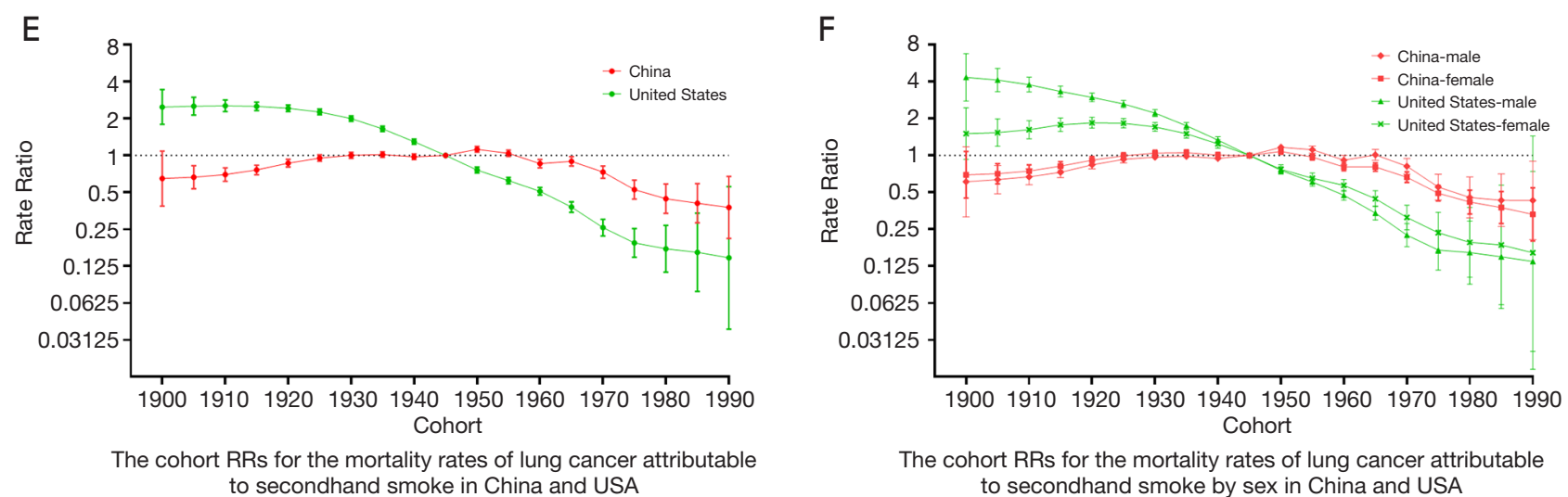

The cohort RRs for the mortality rates of lung cancer attributable to secondhand smoke by sex in China and USA

Figure 5 The age-period-cohort results in the mortality rate of lung cancer attributable to secondhand smoke for both sexes (by sex) from 1990 to 2017.

disease. Therefore, treatment strategies should be focused on older age groups to achieve better control outcomes.

For lung cancer attributable to TE, the period RRs in the USA consistently indicated a downward trend from 1990 to 2017. The United States Congress passed the Federal Cigarette Labeling and Advertising Act and the Cigarette Act in 1965 and 1969, respectively. Also, the Family Smoking Prevention and Tobacco Control Act, passed in the USA in 2007, was more stringent than the WHO's Framework Convention on Tobacco Control (FCTC). Meanwhile, the period RRs in China indicated an upward trend overall, although they declined in the period between 2005 and 2010. China, a signatory to the FCTC (which became operational in 2006), implemented restrictions on the packaging, advertising, and ingredients of tobacco products. For example, a cigarette with $15 \mathrm{mg}$ of 
tar would be regarded as an unqualified product and thus be prohibited from entering the cigarette market. With the implementation of the FCTC in China, the behavior and awareness of tobacco control among the Chinese population greatly improved, and the smoking rate in public places declined, which might explain the decrease in period RRs in China for TE in 2005-2010. However, the China Adult Tobacco Survey Report [2015] found that the smoking rate in China was still high and the rate of exposure to secondhand smoke remained unchanged (18). This situation showed that tobacco control measures in China were insufficient, which may explain the increase in the period RRs for TE. Considering its large population, China must remain vigilant about $\mathrm{TE}$.

The cohort RRs reflect the effects of socioeconomic status changes or different risk factors on people born in different years. The cohort RRs in the USA decreased significantly in the 1900 to 1990 birth cohorts, while those in China increased in the 1900 to 1950 birth cohort, and subsequently decreased in the 1965 to 1990 birth cohort. The cohort RR in China in 1965 was similar to that of the USA in 1935, and the downward trend from 1965 to 1990 in China was similar to that from 1935 to 1960 in the USA. There appears to be a gap of approximately 30 years between the two countries. The USA began enacting legislation regarding tobacco controls in 1965 , while China only began in 2006; again, a gap of about 30 years. The evidence above suggests that lung cancer mortality attributable to smoking declines following the implementation of tobacco control policies by governments. Although the current tobacco control policy in China has been effective, China should learn from the advanced tobacco controls experiences in other countries and establish a comprehensive tobacco control system in accordance with its own national conditions to continue reducing its lung cancer burden.

People smoke for a variety of reasons, including tobacco addiction, level of education, family atmosphere, and social factors. Compared to the USA, social and etiquette smoking for Chinese people is significant, especially in young and middle-aged smokers. This presents an opportunity to provide people with psychological and behavioral advice, particularly for those individuals with social needs, encouraging them to be more confident in refusing cigarettes that are offered to them. Gradually shifting traditional psychology and habits will be beneficial in reducing national smoking rates and increasing the age of first-use of cigarettes. Also, tobacco control measures in China are primarily aimed at the operators of public/ business areas, while punishment measures for smokers are relatively limited compared to the USA, which will likely lead to a slower effect of tobacco controls in China (19). Furthermore, in China, females aged 60 years and males aged 40 years had the highest smoking rates among the population (20), which may be due to the longer life expectancy and lower education level of elderly Chinese women. This indicates that China should not neglect the psychological and living habits of elderly female smokers when formulating tobacco control policy, especially considering that the lung cancer burden attributable to TE in China's elderly population is significantly higher than that in the USA (Figures $3 A, B$ and $5 A, B$ ). Moreover, a large number of studies report that socioeconomic status affects cancer mortality, and may result in delayed detection of cancer and variations in the quality of treatment and health behaviors (including therapeutic compliance) (21-23). For instance, occupation, which largely determines the income of workers and is one of the representative indicators of socioeconomic status, has been recognized as a basic social determinant of health, affecting the incidence and mortality of cancer (24). In Korean and Japanese studies, significant disparities in cancer survival were found among different occupation types $(23,24)$. Since China's economic reform and opening-up, socioeconomic levels in the country have improved substantially, and health awareness has been enhanced among the population, which is one of the reasons for the decline in the lung cancer mortality rate since 1965 .

GBD 2017 could provide sufficient data containing age- and sex-specific all-cause and cause-specific mortality to reduce the possibility of misclassification of outcomes. Based on the effects of age-period-cohort analysis of the temporal trends of lung cancer mortality attributable to TE, and a comprehensive comparison between China and the USA, we found that the risk of death from lung cancer attributable to TE was higher in China than in the USA. If the low smoking age and poor tobacco control measures in China are not improved, death from lung cancer caused by TE will result in enormous economic burdens, especially considering China's population (4).

Some limitations of this study should be noted. Firstly, although the GBD 2017 underwent numerous modifications and adjustments to its data sources, collation, and evaluation methods to improve its data quality and comparability, avoiding bias is difficult. For example, one such bias is that various ways to adjust for mortality data 
are reported by different countries, and thus, the integrity and accuracy of the data in our study was inevitably and unavoidably affected. That being said, it is important to note that using the GBD database to show the longterm trends will not have had an intolerable impact to the present study and minimally affects changes to the longterm trends (25-27). Secondly, the accuracy of data for lung cancer mortality attributable to smoking depends on the establishment and improvement of disease monitoring systems. Chinese data from early years may be inaccurate, which presents a further limitation of our study. Thirdly, the smoking initiation age in China is 20.2 years, and is gradually approaching that of the USA (17.2 years). In other words, the smoking population is gradually becoming younger (28). However, since the GBD 2017 did not contain the period and age intervals data, which should be fixed and equal in the APCM, we did not analyze the lung cancer mortality attributable to smoking in the $0-30$ age group or lung cancer mortality attributable to secondhand smoke in the $0-25$ age group. Therefore, additional studies are required to analyze the lung cancer mortality attributable to TE, especially in the $0-30$ age group. Lastly, for the APC model, despite the non-bias, validity, asymptotic features, and superior estimation ability of the IE method, limitations nonetheless exist. For instance, the parameter estimates generated using this method are not intuitive, the theory behind this method is complicated, and the actual meaning of parameter estimates could not be explained $(29,30)$.

In conclusion, tobacco control strategies have been proven effective in the prevention of lung cancer across a range of different environments. Based on the above findings, China should endeavor to implement targeted tobacco control strategies to reduce the effect of TE on lung cancer mortality over the coming years, which will help to reduce its lung cancer burden.

\section{Acknowledgments}

AME Editing Service (http://editing.amegroups.com) helped with the language finishing of this paper.

Funding: None.

\section{Footnote}

Reporting Checklist: The authors have completed the MDAR checklist. Available at: http://dx.doi.org/10.21037/atm-20996
Conflicts of Interest: All authors have completed the ICMJE uniform disclosure form available at: http://dx.doi. org/10.21037/atm-20-996). The authors have no conflicts of interest to declare.

Ethical Statement: The authors are accountable for all aspects of the work in ensuring that questions related to the accuracy or integrity of any part of the work are appropriately investigated and resolved. The study was conducted in accordance with the Declaration of Helsinki (as revised in 2013). All our research data are obtained from GBD 2017, the website was http://ghdx.healthdata.org/ gbd-results-tool. GBD 2017 was publicly available for free use. The ethical approval was not required.

Open Access Statement: This is an Open Access article distributed in accordance with the Creative Commons Attribution-NonCommercial-NoDerivs 4.0 International License (CC BY-NC-ND 4.0), which permits the noncommercial replication and distribution of the article with the strict proviso that no changes or edits are made and the original work is properly cited (including links to both the formal publication through the relevant DOI and the license). See: https://creativecommons.org/licenses/by-nc-nd/4.0/.

\section{References}

1. Bray F, Ferlay J, Soerjomataram I, et al. Global cancer statistics 2018: GLOBOCAN estimates of incidence and mortality worldwide for 36 cancers in 185 countries. CA Cancer J Clin 2018;68:394-424.

2. Chen W, Zheng R, Baade PD, et al. Cancer statistics in China, 2015. CA Cancer J Clin 2016;66:115-32.

3. Siegel RL, Miller KD, Jemal A. Cancer statistics, 2019. CA Cancer J Clin 2019;69:7-34.

4. GBD 2015 Tobacco Collaborators. Smoking prevalence and attributable disease burden in 195 countries and territories, 1990-2015: a systematic analysis from the Global Burden of Disease Study 2015. Lancet 2017;389:1885-906.

5. Ahluwalia IB, Smith T, Arrazola RA, et al. Current Tobacco Smoking, Quit Attempts, and Knowledge About Smoking Risks Among Persons Aged $\geq 15$ Years - Global Adult Tobacco Survey, 28 Countries, 2008-2016. MMWR Morb Mortal Wkly Rep 2018;67:1072-6.

6. Wang GZ, Zhang L, Zhao XC, et al. The Aryl hydrocarbon receptor mediates tobacco-induced PDL1 expression and is associated with response to 
immunotherapy. Nat Commun 2019;10:1125.

7. Steliga MA, Dresler CM. Epidemiology of lung cancer: smoking, secondhand smoke, and genetics. Surg Oncol Clin N Am 2011;20:605-18.

8. de Groot P, Munden RF. Lung cancer epidemiology, risk factors, and prevention. Radiol Clin North Am 2012;50:863-76.

9. Zhou M, Wang H, Zeng X, et al. Mortality, morbidity, and risk factors in China and its provinces, 1990-2017: a systematic analysis for the Global Burden of Disease Study 2017. Lancet 2019;394:1145-58.

10. Shi JF, Liu CC, Ren JS, et al. Economic burden of lung cancer attributable to smoking in China in 2015. Tob Control 2020;29:191-9.

11. World Health Organization. WHO global report on trends in prevalence of tobacco smoking 2000-2025. 2018.

12. Global Health Data Exchange. GBD Results Tool. Available online: http://ghdx.healthdata.org/gbd-resultstool. Accessed June 102019.

13. Sommer G, Schindler M, Redmond S, et al. Temporal trends in incidence of childhood cancer in Switzerland, 1985-2014. Cancer Epidemiol 2019;61:157-64.

14. Yang GH, Ma JM, Liu N, et al. Smoking and passive smoking in Chinese, 2002. Zhonghua Liu Xing Bing Xue Za Zhi 2005;26:77-83.

15. Hoang Anh PT, Thu le T, Ross H, et al. Direct and indirect costs of smoking in Vietnam. Tob Control 2016;25:96-100.

16. Sung HY, Chang LC, Wen YW, et al. The costs of smoking and secondhand smoke exposure in Taiwan: a prevalence-based annual cost approach. BMJ Open 2014;4:e05199.

17. Amarasinghe $\mathrm{H}$, Ranaweera $\mathrm{S}$, Ranasinghe $\mathrm{T}$, et al. Economic cost of tobacco-related cancers in Sri Lanka. Tob Control 2018;27:542-6.

18. Chinese Center for Disease Control and Prevention. China Adult Tobacco Survey Report 2015. 2015.

19. Jiang Y, Xiao Q. Epidemic and control status on tobacco in China. Occup and Health 2018;34:997-9.

20. Xue $X$, Jiang G, Li W, et al. Status quo on smoking and related change pattern of smoking related behavior in past 20 years in urban residents in Tianjin. Chin J Epidemiol

Cite this article as: Wu X, Zhu B, Xu S, Liu Y, Bi Y, Zhou B. A comparison of the burden of lung cancer attributable to tobacco exposure in China and the USA. Ann Transl Med 2020;8(21):1412. doi: 10.21037/atm-20-996
2019:1095-8.

21. Chien LH, Tseng TJ, Tsai FY, et al. Patterns of agespecific socioeconomic inequalities in net survival for common cancers in Taiwan, a country with universal health coverage. Cancer Epidemiol 2018;53:42-48.

22. Zaitsu M, Kim Y, Lee HE, et al. Occupational class differences in pancreatic cancer survival: A populationbased cancer registry-based study in Japan. Cancer Med 2019;8:3261-8.

23. Lee HE, Zaitsu M, Kim EA, et al. Occupational Class and Cancer Survival in Korean Men: Follow-Up Study of Nation-Wide Working Population. Int J Environ Res Public Health 2020;17:303.

24. Zaitsu M, Kaneko R, Takeuchi T, et al. Occupational inequalities in female cancer incidence in Japan: Hospitalbased matched case-control study with occupational class. SSM Popul Health 2018;5:129-37.

25. Lancet T. GBD 2017: a fragile world. Lancet 2018;392:1683.

26. Afshin A, Sur PJ, Fay KA, et al. Health effects of dietary risks in 195 countries, 1990-2017: a systematic analysis for the Global Burden of Disease Study 2017. Lancet 2019;393:1958-72.

27. James SL, Abate D, Abate KH, et al. Global, regional, and national incidence, prevalence, and years lived with disability for 354 diseases and injuries for 195 countries and territories, 1990-2017: a systematic analysis for the Global Burden of Disease Study 2017. Lancet 2018;392:1789-858.

28. Giovino GA, Mirza SA, Samet JM, et al. Tobacco use in 3 billion individuals from 16 countries: an analysis of nationally representative cross-sectional household surveys. Lancet 2012;380:668-79.

29. Fu W, Land K. The Intrinsic Estimator for Age-PeriodCohort Analysis: What It Is and How to Use It. American Journal of Sociology 2008;113:1697-736.

30. Liu X, Yu C, Bi Y, et al. Trends and age-period-cohort effect on incidence and mortality of prostate cancer from 1990 to 2017 in China. Public Health 2019;172:70-80.

(English Language Editors: A. Kassem and J. Reynolds) 